\section{《內分泌、代謝及㹂脏病学术 会議資料选編》}

\author{
中华医学会編 \\ 上海科学技术出版社出版
}

1964 年 5 月份中华医学会在广州召开內分泌、 代谢及肾肚病学术会议，这次会议共收到论文 378 篇，井从中选出“內分泌研究发展的方向”等 5 篇综 述性的文章及“助阳药与滋阴药在內分䎵和 肾脏病 方面的实验研究”和“中华医学会內分泌、代谢及肾 脏病学术会议总结报告”等文印成专集，以㗽 读 者。在本集中还附录了 1962-1964 年北京 生 理学 会举行的內珎泌学术貄座三篇论文。

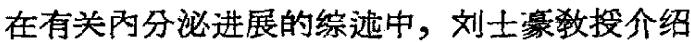
了內分泌学中的调节与控制、激素的生物合成、激 素的运转机制、激素的化学结构与功能的关系、內 分泌的免疫学、激素的作用机制等六个问题和它们 在国际上现代发展的状况。其他作者对地方甲状腺 肿与地方克汀病、类固醇激素的研究动向、激素与 肿㾿、雄激素等问题，也作了较系统的阐述。

北京医学院王叔咸授在“有关蛋白质代 谢的 若干问题”一文中，综述了由于蛋白质代谢的研究
工作中应用了超速离心机、电子显微镜、电泳、色 昙分析、同位素示踪等的研究新方法后，人们对蛋 白质代谢过程及机制问题上的观点有所提高的情 况，作者井根据这些观点提出今后临床工作应当注 意研究的几个问题。

在“助阳药和滋阴药在內分泌和肾胜病方面的 实验研究”一文中，用现代的实验研究方法来研 究租国医学部门的成就，未介绍了上海第二医学院 的药理、內科二个研组和上海市高血压研究所, 从1959 年以来，在临床和动物试验方面研 究助阳 药和滋阴药在阿分泌与肾脏病所取得的成果, 分析 了祖国医学中某些科学原理。

中华医学会內分泌、代谢及肾脏病学术会议总 结摘要中总结了我国解放后在垂体肾上腺、性腺和 乳腺的研究工作中所取得的成果和对甲状腺、糖尿 病、甲状旁腺病及肾脏病等方面治愈的情况。并指 出了这方面的研究工作中还存在着若干国际性的问 题。

在本总结之后还附有关于肾小球肾炎的临床分 型、慢性肾小球肾炎的治疗及治愈标准的参考意见 和关于肾盂肾炎的诊断、治疗及治愈标准的参考意 见的两个附件。

本书可供內分珌、蛋白质代谢及肾胜病的研究 工作者及监床大夫淁考之用。

[馬月英]

\title{
更正
}

本刊 1965 年 10 月号第 847 页右栏第 10 行 “为什么会这样呢？主 要因为"等字应删去。特此更正。 